\title{
La anemia por deficiencia de hierro: estrategias de la OPS/OMS para combatirla
}

W ilma B. Freire, Ph.D. ${ }^{1}$

\section{Freire WB. \\ La anemia por deficiencia de hierro: estrategias de la OPS/OMS para combatirla. Salud Publica Mex 1998;40:199-205.}

\section{Resumen}

La anemia por deficiencia de hierro es uno de los problemas nutricionales de mayor magnitud en el mundo. A pesar de conocer su etiología y tener a disposición el conocimiento de cómo enfrentarla y de saber que las intervenciones son de bajo costo, aún no se ha podido superar este problema. Este documento parte de una estimación de la magnitud del problema y plantea los elementos necesarios para el diseño, la ejecución y la medición del impacto de la suplementación con hierro y la fortificación, como las intervenciones más efectivas para disminuir considerablemente la dimensión de la anemia por falta de hierro. Propone una lista de pasos a seguir previos a la elaboración de un proyecto y recomienda una serie de elementos a considerar en ello. Define, asimismo, los aspectos que se deben incluir en una propuesta de fortificación y en otra de suplementación. El documento concluye con un listado de actividades complementarias que la 0 rganización Panamericana de la Salud/ O rganización Mundial de la Salud ofrece dentro de su paquete de cooperación técnica.

Palabras clave: anemia; micronutrientes; deficiencia de hierro; Mexico
Freire WB.

Iron deficiency anemia:

PAHO/WHO strategies to fight anemia.

Salud Publica Mex 1998;40:199-205.

\section{A bstract}

Iron deficiency anemia is among the greatest nutritional problems in the world. Although its etiology is understood and intervention at low cost is available, the problem persists. The present review begins with a general estimate of the dimensions of the problem. It suggests the necessary elements for the design, implementation, and measurement of the impact of iron supplementing and fortification as the most effective forms to intervene and diminish iron deficiency anemia. Several preliminary steps are proposed previous to the preparation of a project and several recomendations are made to be included in a project for fortification and iron supplementing. A list of complementary activities offered by PAHO /W HO as part of the package of technical cooperation is included.

Key words: anemia; micronutrients; iron deficiency; Mexico

(1) Cordinadora del Programa de Alimentación y N utrición, O rganización Panamericana de la Salud/O rganización Mundial de la Salud (O PS/O MS).

Fecha de recibido: 10 de julio de 1997 - Fecha de aceptado: 15 de enero de 1998 Solicitud de sobretiros: Dra. W ilma B. Freire. O PS/O MS. 525 23rd. St. N.W.W ashington, D.C.. 20037-2895, USA. 
L a anemia por deficiencia de hierro es uno de los problemas nutricionales de mayor magnitud en el mundo. A pesar de que se conoce tanto su etiología como la forma de enfrentarla y de que las intervenciones son de bajo costo, aún no se ha podido resolver este problema.

El punto de partida de este trabajo es una estimación de la magnitud del problema; aquí se plantean los elementos necesarios para el diseño, la ejecución y la medición del impacto de la suplementación con hierro y la fortificación, como las intervenciones más efectivas para disminuir la cifra de casos de anemia por falta de hierro.

Este tipo de deficiencia se presenta cuando la cantidad de hierro disponible es insuficiente para satisfacer las necesidades individuales; la exposición a una deficiencia prolongada conduce a la anemia. Se estima que más de 2000 millones de personas sufren de deficiencia de hierro y que más de la mitad está anémica; la prevalencia de anemia entre las embarazadas, los infantes y los menores de dos años en los países en desarrollo supera el 50\%; entre niños en edad prescolar y entre mujeres en edad fértil es un poco más baja, pero siempre de magnitudes importantes. ${ }^{1-3}$

La anemia en niños e infantes está asociada con retardo en el crecimiento y en el desarrollo cognoscitivo, así como con una resistencia disminuida a las infecciones. En los adultos, la anemia produce fatiga y disminuye la capacidad de trabajo físico. En las embarazadas se asocia con el bajo peso al nacer y un incremento en la mortalidad perinatal. La deficiencia de hierro inhibe la habilidad de regular la temperatura cuando hace frío y altera la producción hormonal y el metabolismo, afectando los neurotransmisores y las hormonas tiroideas asociadas con las funciones musculares y neurológicas, reguladoras de la temperatura. ${ }^{4-12}$

Mientras la deficiencia de hierro afecta el desarrollo cognoscitivo en todos los grupos de edad, los efectos de la anemia en la infancia y durante los primeros años de vida son irreversibles, aun después de un tratamiento. Al cumplir su primer año de vida, $10 \%$ de los infantes en los países desarrollados, y alrededor de 50\% en los países en desarrollo, están anémicos; esos niños sufrirán retardo en el desarrollo psicomotor, y cuando tengan edad para asistir a la escuela, su habilidad vocal y su coordinación motora habrán disminuido significativamente. ${ }^{1}$

\section{Situación en el continente americano}

La información disponible al respecto indica que en el continente americano aproximadamente 94 millones de personas sufren de anemia ferropénica, ${ }^{3}$ y que las mujeres embarazadas y los niños pequeños presentan las más altas prevalencias. Se estima que por cada anémico por lo menos una persona más es deficiente de hierro, lo cual se traduce en un problema de enorme magnitud. La situación es extremadamente grave en algunas áreas, como por ejemplo en los países del Caribe donde se notifican prevalencias del orden de $60 \%$ entre las mujeres embarazadas.

Pocos países cuentan con información detallada acerca de la prevalencia de anemia. Así, Ecuador, por ejemplo, notificó una prevalencia nacional de $70 \%$ en los niños de 6-12 meses de edad, y de $45 \%$ en aquellos de 12-24 meses. ${ }^{13}$ Cuba informó que $64 \%$ de los niños de 1-3 años ${ }^{14}$ sufren de anemia; en Misiones, Argentina, la prevalencia es de $55 \%$ en los niños de 9-24 meses, ${ }^{15}$ y en México, de 50.7\% en una muestra de 152 niños cuya edad oscilaba entre los 6 y los 36 meses. ${ }^{16}$ En todos los estudios de caso se indica que la población más afectada es la de los recién nacidos de bajo peso, los menores de dos años y las mujeres embarazadas. ${ }^{13,17}$

\section{Acuerdos Internacionales}

En respuesta a la abundante evidencia de que la deficiencia de hierro es un problema de grandes magnitudes y con consecuencias ilimitadas en la población, más de 170 jefes de Estado se comprometieron, en la Cumbre Mundial en Favor de la Infancia (1990), a reducir en un tercio la prevalencia de anemia en embarazadas hasta el año 2000. Del mismo modo, en la Conferencia Internacional de Nutrición (1992) se adoptó el compromiso de luchar por reducir las altas prevalencias de anemia, que afectan particularmente a los niños y a las mujeres embarazadas. Como agencia especializada de las Naciones Unidas, la Organización Panamericana de la Salud/Organización Mundial de la Salud (OPS/OMS) adoptó como prioritario el compromiso de combatir esta deficiencia en todo el continente americano. ${ }^{19}$

\section{Actividades}

La OPS/OMS ha propuesto la realización, desde 1996 hasta el año 2000, de una serie de actividades regionales, nacionales y locales que contribuirán a reducir sustancialmente las anemias ferropénicas. El plan para llevarlas a cabo será evaluado para determinar el impacto de la cooperación técnica brindada por la OPS/ OMS en está área de trabajo. ${ }^{19}$

Desde la perspectiva regional periódicamente se reúne un grupo de técnicos y expertos representantes de los diversos organismos internacionales de coo- 
peración, de las organizaciones no gubernamentales (ONG) y de las agencias donantes, para analizar el avance de las actividades, compartir las experiencias y la información, y revisar el avance de los acuerdos. En este grupo participan el Fondo de las Naciones Unidas para la Infancia (UNICEF), la Agency for International Development/Opportunities for Micronutrient Intervention, el Banco Mundial y Mothercare, entre otros.

$\mathrm{Al}$ servicio de la región, se puso una hoja electrónica en el World Wide Web, y a través del Internet, un servidor de listas de correo, para ofrecer información periódicamente. Asimismo, por ese medio el Instituto de Tecnología de Alimentos y Nutrición de Chile (INTA) ofrece consultoría gratuita sobre diferentes temas relacionados con el problema de la deficiencia de hierro y la anemia.

Se organizó una red de consultores temporales en las diferentes líneas de cooperación y se puso a la disposición de los diversos países, con el objeto de cubrir las necesidades de cooperación. Del mismo modo, también se han preparado guías metodológicas que pueden utilizarse localmente en el desempeño de las diferentes actividades.

\section{A reas de Cooperación}

Con el fin de aminorar la cifra de casos de deficiencia de hierro y de anemia, la OPS/OMS recomienda que los países pongan en ejecución, en forma paralela, un conjunto de intervenciones cuyo impacto sea en el corto, el mediano y el largo plazo. Esta propuesta parte de un enfoque integral tanto del problema de las anemias como de su solución, y su ejecución exige la participación del sector salud y de otros sectores públicos y privados en sus campos respectivos de acción. Estas intervenciones se resumen en la fortificación de un alimento de consumo masivo, de bajo costo y disponible en el mercado, y en la entrega de suplementos con hierro a mujeres embarazadas y a niños menores de dos años. Ambas intervenciones deben ir acompañadas de actividades de promoción a través de los medios de comunicación, vigilancia epidemiológica y sistemas de garantía de calidad, para hacer un seguimiento del programa y medir el impacto.

\section{Medidas}

A fin de contar con los elementos necesarios para formular una propuesta integral, la OPS/OMS recomienda iniciar las actividades con un análisis de la situación que incluya los siguientes aspectos.

\section{Nivel de deficiencia de hierro y anemia en el país}

La OPS/OMS propone que se recopilen los datos pertinentes para determinar la magnitud, la severidad, y la distribución geográfica por sexo de la anemia por falta de hierro, a fin de establecer un punto de referencia antes de iniciar los programas de suplementación y fortificación. Del mismo modo, es necesario recopilar información sobre las características de la dieta, la presencia de otras deficiencias y la dimensión de infestación parasitaria. Diversos países carecen de información que permita estimar la magnitud y distribución del problema; si éste es el caso y no se puede construir un punto de referencia que sustente el diseño de la intervención y las evaluaciones subsiguientes, será necesario diseñar una propuesta para obtener dicha in formación. La OPS/OMS considera que no es necesario hacer un estudio sofisticado y costoso para la construcción de tal referencia. Para ello recomienda la ejecución de un estudio rápido que permita obtener un mínimo de información para sustentar las intervenciones que se llevarán a cabo. Este tipo de estudio puede formar parte de las encuestas periódicas que se llevan a cabo en diversos países, como por ejemplo las encuestas sobre fecundidad, presupuestos familiares y empleo, entre otras.

En este trabajo no se discuten las técnicas de muestreo ni los indicadores o la metodología para recolectar la información. En caso de que algún país solicite cooperación técnica para todo o parte del diseño del estudio que constituirá el punto referencial, la OPS/ OMS brindará apoyo inmediato por medio de su personal o de la red de consultores.

\section{Análisis de la capacidad industrial para la fortificacion de productos}

Una vez identificados los posibles vehículos que serán fortificados, es necesario analizar la capacidad industrial de las plantas que procesan los alimentos en términos de los equipos de laboratorios, del grado de formación técnica del personal, y del sistema de empaquetado y distribución, para conocer sus características y, de ser necesario, determinar el tipo de adecuaciones que requiere la planta para producir el alimento fortificado.

\section{Análisis del sistema de servicios de salud}

Si se decide complementar con hierro a mujeres embarazadas y niños menores de dos años, es necesario identificar las condiciones de los servicios de salud en 
términos de calidad y cobertura, el grado de conocimientos del personal en cada nivel de prestación y la capacidad de almacenamiento del suplemento, entre otros aspectos. Del mismo modo, se deben anticipar los posibles problemas y cuellos de botella que entorpezcan el éxito de la intervención, y diseñar formas para disminuir su impacto negativo en las actividades de suplementación

\section{Capacidad técnica}

Para determinar el apoyo técnico que necesita cada país es necesario evaluar la capacidad técnica local que podría asumir las diferentes responsabilidades (p.e. gerencia y gestión; adecuación de la maquinaria industrial para la fortificación; diseño del sistema de vigilancia; diseño del sistema de garantía de calidad; actividades de mercadeo y comunicación; capacitación del personal de salud; etc.). Esta actividad permitirá definir cuándo y qué tipo de cooperación técnica se puede brindar.

La OPS/OMS tiene la posibilidad de brindar la cooperación técnica para el diseño y la ejecución de un programa de fortificación y suplementación. Para lograr este fin, dicho organismo puede movilizar los recursos humanos para responder a las necesidades técnicas y específicas de cooperación, por medio de su red de consultores o en coordinación con otras agencias de cooperación.

\section{Estudio de factibilidad}

Para garantizar la viabilidad de un programa nacional se debe establecer la viabilidad económica de la propuesta e identificar a los posibles contribuidores, donantes o prestamistas, privados o del gobierno, nacionales e internacionales. Esta actividad tiene por objeto lograr la participación de instituciones nacionales de gobierno y privadas, de las ONG y de organismos internacionales para garantizar el apoyo técnico $\mathrm{y}$ financiero necesario.

\section{Compromiso político}

Un elemento esencial para la viabilidad y el éxito de un programa de fortificación o suplementación, es el compromiso adquirido por el nivel político de decisión, en términos de liderazgo, asignación de fondos y otros recursos, de tal manera que quede garantizado que las máximas autoridades asumen el compromiso de ejecutar el programa. A este respecto, la OPS/OMS realiza una intensa labor de abogacía, con el fin de lograr el compromiso político y técnico, ya que sin éste es imposible que una propuesta, por bien concebida que esté, llegue a ponerse exitosamente en marcha.

\section{Elaboración del proyecto}

A partir del análisis de la situación, procede la elaboración de la propuesta que constituye el marco de ejecución de cada intervención, sea ésta la fortificación de un alimento de consumo masivo y de bajo costo, o bien, la suplementación con hierro a mujeres embarazadas y a niños pequeños, o ambas. La propuesta debe desarrollar componentes de promoción, comunicación, educación, un sistema de vigilancia epidemiológica nutricional y de garantía de calidad. En este documento no se busca entrar en el detalle específico de cada intervención, pero cabe observar que el desarrollo de cada intervención involucra habilidades específicas y requiere de la contribución de varias disciplinas.

En el cuadro I se presentan los elementos que debe incluir una propuesta integral para combatir la anemia por falta de hierro. En ella se especifican los componentes de la propuesta. Es necesario que al elaborar la propuesta, se desarrolle cada componente con suficiente detalle de tal manera que se facilite su inmediata ejecución.

En el cuadro II se presentan los elementos de una propuesta de fortificación; cada componente debe desarrollarse de acuerdo con la situación del país. Por ejemplo, se recomienda que la fortificación se haga en alimentos de consumo masivo y de bajo costo, pero también puede optarse por fortificar alimentos dirigidos a ciertos grupos de población como los escolares. Asimismo, es importante adoptar una ley que defina claramente los derechos y las obligaciones que corresponden a cada sector involucrado.

Es también esencial que se definan con claridad todos los componentes de un sistema de garantía de calidad desde el inicio de la producción hasta la distribución y el consumo. Todo esto no hace sino reafirmar la necesidad de que al elaborar la propuesta de fortificación se detalle en la forma más explícita posible cada uno de los componentes y se definan las responsabilidades que le toca asumir a cada sector participante.

El cuadro III muestra los componentes de un programa de suplementación; en relación con esta intervención es extremadamente útil disponer de información sobre la infraestructura, la calidad y la cobertura de los servicios de salud, así como sobre el nivel de información que tiene el personal de salud y la percepción que los posibles usuarios tienen sobre la administración de 


\section{Cuadro I}

Componentes de Un PLAN INTEgral PARA disminuir LA ANEMIA FERROPÉNICA

\begin{tabular}{|c|c|}
\hline A nálisis de la situación & $\begin{array}{l}\text { Compromiso político } \\
\text { Situación clínica y epidemiológica de la anemia } \\
\quad \text { por falta de hierro } \\
\text { Programas existentes. Marco institucional } \\
\text { Beneficios esperados }\end{array}$ \\
\hline $\mathrm{O}$ bjetivos & $\begin{array}{l}\text { Generales } \\
\text { Específicos } \\
\text { O peracionales } \\
\text { De impacto }\end{array}$ \\
\hline Estrategias & $\begin{array}{l}\text { Fortificación } \\
\text { Suplementación } \\
\text { Promoción, comunicación, educación } \\
\text { Vigilancia epidemiológica nutricional }\end{array}$ \\
\hline Plan de acción & $\begin{array}{l}\text { O rganización gerencial } \\
\text { Recolección de información para construir el } \\
\quad \text { punto de referencia } \\
\text { Identificación de alimentos de consumo masivo } \\
\text { A nálisis de viabilidad de la suplementación } \\
\text { Definición de población objetivo } \\
\text { A nálisis de viabilidad de la fortificación }\end{array}$ \\
\hline $\begin{array}{l}\text { Actividades suplemen- } \\
\text { tarias de apoyo: }\end{array}$ & $\begin{array}{l}\text { A bogacía } \\
\text { Coordinación interagencial local } \\
\quad \text { e internacional } \\
\text { Movilización de recursos } \\
\text { Desarrollo de recursos humanos } \\
\text { Promoción de la investigación }\end{array}$ \\
\hline Gerencia & $\begin{array}{l}\text { Coordinación interagencial } \\
\text { Vinculación con otros sectores } \\
\text { Vinculación con otros programas } \\
\text { Papel de las instituciones especializadas } \\
\text { N egociación con diversas fuentes de } \\
\quad \text { financiamiento }\end{array}$ \\
\hline
\end{tabular}

Adaptado de W HO, $1994^{2}$

hierro para el tratamiento de la anemia. Esta información permite determinar los puntos críticos que deben corregirse y facilita la toma de decisiones adecuadas y oportunas para garantizar que la suplementación cumpla con sus objetivos. Dado que en la mayoría de los países la cobertura de los servicios de atención prenatal es baja, se debe prever la adopción de nuevas formas de captación precoz y seguimiento a las embarazadas y menores de dos años que reciben el suplemento, utilizando otras vías como la Iglesia, las ONG y las escuelas, entre otras.

Para el monitoreo y la evaluación de cada programa de suplementación es necesario diseñar un sistema de detección temprana. Del mismo modo, se requiere de manuales de operación para el personal de campo que instruyan con detalle acerca de cómo identificar a

\section{Cuadro II \\ Componentes de una PROPUESTA de Fortificación}

Componentes

\begin{tabular}{ll} 
Razones para la fortificación & $\begin{array}{l}\text { Ventajas } \\
\text { Oportunidades } \\
\text { Limitaciones }\end{array}$ \\
\hline Población objetivo & $\begin{array}{l}\text { Población total } \\
\text { Embarazadas } \\
\text { Menores de dos años } \\
\text { Adolescentes }\end{array}$ \\
\hline Selección de vehículo & De consumo masivo y de bajo costo \\
\hline Definición de responsabilidades & $\begin{array}{l}\text { Legislativo } \\
\text { Garantía de calidad } \\
\text { Vigilancia epidemiológica } \\
\text { Financiamiento }\end{array}$ \\
\hline Infraestructura & Infraestructura industrial \\
& Tecnología \\
& Sistema de garantía de calidad \\
\hline Selección de fortificante & Costo \\
& Disponibilidad y acceso \\
& Efecto en la preparación doméstica \\
& Aceptabilidad \\
\hline Eromoción & Abogacía \\
& Mercado social \\
& Educación, comunicación \\
\hline Monitoreo del proceso & Industrial \\
& De consumo \\
\hline & $\begin{array}{l}\text { Población en riesgo } \\
\text { Población total } \\
\text { Sustentabilidad }\end{array}$ \\
\hline
\end{tabular}

Adaptado de W HO, $1994^{2}$

la población objetivo, conducir la evaluación, identificar riesgo y desarrollar actividades de educación, así como de registro e interpretación de la información. Los manuales también deben indicar los instrumentos que son necesarios y cómo deben utilizarse. Finalmente, la propuesta tiene que desarrollar los mecanismos que deben emplearse para llegar a la población objetivo con el suplemento, hacer el seguimiento al consumo e identificar dónde puede obtener los suplementos y cómo puede almacenarlos.

En relación con las actividades de educación, promoción y comunicación, será necesario definir los temas de los mensajes, el costo y la frecuencia de transmisión, así como las actividades complementarias de promoción que deberán realizarse cara a cara.

Antes de diseñar un sistema de vigilancia que sea efectivo, es necesario revisar los sistemas vigentes de recolección de datos e investigar las posibles vías por medio de las cuales también se puede recolectar in- 


\section{Cuadro III \\ Componentes de una propuesta DE SUPLEMENTACIÓN}

Componentes

- Definición del programa de acción

- Definición de población objetivo: embarazadas, menores de dos años, escolares, adolescentes

- Número de población objetivo que será beneficiada

- Definición del suplemento, dosis

- Definición de mecanismos operativos: captación precoz, monitoreo de actividades

- Diseño de instrumentos

- Definición y aplicación de la prueba de campo

- Capacitación de personal

- Preparación de manuales y estándares

- Monitoreo de proceso

- Evaluación de impacto

formación periódica, como por ejemplo las encuestas periódicas de fecundidad, las de empleo u otras que disponen de diseños muestrales maestros y a las que se les pueden agregar módulos especiales sobre nutrición. Para que un sistema de vigilancia tenga éxito es necesario que garantice la recolección periódica de información confiable, con diseños de muestra que aseguren la representatividad de la población objeto de la vigilancia.

Como se desprende de lo anterior, el conjunto de actividades de un programa integral conducente a reducir las altas prevalencias de anemia por falta de hierro, no puede estar en manos de un solo especialista o institución. Por ello, se recomienda la conformación de grupos técnicos de trabajo, apoyados por especialistas que residan en el país o que sean trasladados temporalmente para cumplir tareas concretas. Mediante esta forma de trabajo la OPS/OMS puede brindar cooperación técnica específica tanto para la elaboración de la propuesta como para la movilización de recursos.

\section{Actividades complementarias de apoyo de la OPS/OMS}

\section{Coordinacion interinstitucional y movilizacion de recursos}

De acuerdo con la OPS/OMS, es esencial establecer agendas de trabajo coordinadas entre los organismos internacionales de cooperación, las instituciones locales involucradas y los ONG. Esta forma de trabajo garantiza el uso óptimo de los recursos y una excelente participación de todas las instancias involucradas. De esa manera, es necesario promover activamente los esfuerzos de cooperación entre organismos locales e internacionales para reducir las altas prevalencias de anemia por falta de hierro.

\section{Informacion, generación y diseminacion de informacion}

Disemina información y promueve la elaboración de documentos técnico-normativos relevantes al tema. Para garantizar esta actividad, elabora su propio material, pero también utiliza el de otras instituciones. Para lograr una óptima difusión de la información, la OPS/OMS ha puesto al servicio de la región su página electrónica en el World Wide Web y su servidor de lista de correo.

\section{Desarrollo de recursos humanos}

Organiza talleres y seminarios para la elaboración de propuestas, para gerencia, vigilancia, control de calidad y otras actividades relacionadas con garantía de calidad, planificación de programas, diseño y ejecución. A través de estos seminarios se promueve el intercambio internacional de experiencias, así como la cooperación técnica horizontal y se desarrollan habilidades específicas en los diferentes temas.

\section{Promocion de la investigacion}

Promueve y apoya la investigación científica cuyo resultado puede tener utilización inmediata en la ejecución de actividades, locales, nacionales y regionales. La investigación se concibe como una actividad necesaria para mejorar, modificar u optimizar las intervenciones.

\section{Cooperación técnica}

Proporciona a los países cooperación técnica para el diseño y la ejecución de programas dirigidos a combatir la deficiencia de hierro y la anemia. Dentro de su línea de trabajo está, además, la abogacía, la promoción de la cooperación interagencial y la diseminación de información; todo encaminado al fortalecimiento institucional de los países. El Instituto de Nutrición de Centro América y Panamá (INCAP) y el Caribbean Food and Nutrition Institute, como centros especializados de la OPS/OMS, realizan actividades similares en sus respectivas áreas de influencia. Adicionalmente, cuenta con una red de consultores temporales que están al servicio de los países para cubrir necesidades técnicas específicas, todo esto bajo la conducción técnica del Programa de alimentación y nutrición en el ámbito regional. 


\section{Referencias}

1. Pollitt E. Malnutrition and infection in the classroom. París: UN ESC O, 1990.

2. W orld Health O rganization. Report of W HO /UN ICEF/Joint Committee on Health Policy, 30th Session. Strategic approach to operationalizing selected end decade goals: reduction of iron deficiciency anemia by one third of the 1990 levels. JCHP30/95/4.5. Ginebra:W HO, 1994.

3. W orld Health 0 rganization. Report of W HO /UN ICEF/UN U consultation on indicators and strategies for iron deficiency and anemia programmer. Draft IDA REP.01. Ginebra: W HO, 1994.

4. Chandra RK, Saraya AK. Impaired immunocompetence associated with iron deficiency. J Pediatr 1975;86:899-902.

5. Gardner GW , Edgerton VR, Senewiratne B et al. Physical work capacity and metabolic stress in subjects with iron deficiency anemia. Am J Clin N utr 1977;30:910-917.

6. Basta S, Soedirman, Karyadi D, Scrimshaw NS. Iron deficiency anemia and the productivity of adult males in Indonesia. Am J C lin N utr 1979;32: 916-925.

7.W alter T,Arrendon S, Stekel AM. Effect of iron therapy on phagocytosis and bacterial activity in neutrophils of iron deficient infants. Am J Clin N utr 1986:44-82.

8. Beard JL, Borel M. Thermogenesis and iron deficiency anemia. N utr Today 1988;23:41-45.

9. Sheshadri S, Gopaldas T. Impact of iron supplementation on cognitive functions in preschool and school age children: The Indian experience. Am J C lin N utr 1989;50(3):675-686.
10. SoemantriAG. Preliminary findings on iron supplementation and learning achievement of rural Indonesian children. Am J C lin N utr 1989;50(3): 698-702.

11. Freire W B, Dirren H, Barclay D. Iron deficiency anemia in Ecuador. En: Hercberg S, Galan P, Dupin H, ed. Recent knowledge on iron and folate deficiencies in the world. París: Collogue IN SEA M, 1990;197:47-54. 12. Lozoff $B$, Jiménez $E$, W olf AW. Long term developmental outcome of infants with iron deficiency. N Engl J Med 1991;325(10):687-695.

13. FreireW B, Dirren H, Mora JO et al. Diagnóstico de la situación alimentaria, nutritional y de salud de la población ecuatoriana menor de cinco años. Q uito, Ecuador: CO N ADE/MSP, 1988.

14. Guy J, Padrón M,Amador M. Prevención y control de la anemia y la deficiencia de hierro en Cuba. Rev Cubana Aliment Nutr 1995;9(1): 52-61.

15. Calvo EV, Guazzo N. Prevalence of iron deficiency in children aged 924 months from a large urban area in Argentina. Am J C lin N utr 1990;52: 534-540.

16. Rosado JL, Bourgers H, Saint-Martin B. Deficiencia de vitaminas y minerales en México: una revisión crítica de información. I. D eficiencia de minerales. Salud Publica Mex 1995;37:130-139.

17. Tufts D, Hass JD, Beard JL, Spielvogel H. Distribution of hemoglobin and functional consequences of anemia in adult males at high altitude.Am J Clin N utr 1985;42:1-11.

18. Freire W B. Hemoglobin as a predictor of response to iron theraphy and its use in screening and prevalence estimates. Am J C lin N utr 1989;50: 1442-1449.

19. O rganización Panamericana de la Salud/O rganización Mundial de la Salud. Plan regional de alimentación y nutrición. W ashington, D.C.: O PS/ O MS, 1997. 